\title{
Triptycene-based Three-Dimensional Covalent Organic Frameworks with stp Topology of Honeycomb Structure
}

\author{
Yuancheng Wang, ${ }^{\mathrm{a},+}$ Chenyu Wu, ${ }^{\mathrm{a},+}$ Wenjia Sun,,${ }^{\mathrm{b}++}$ Qingyan Pan, ${ }^{\mathrm{a}}$ Wenbo Hao, ${ }^{\mathrm{a}}$ Hui Liu, ${ }^{\mathrm{a}}$ Jing Sun, ${ }^{\mathrm{a}}$ \\ Zhibo Li, ${ }^{*, a}$ Junliang Sun, ${ }^{*, b}$ and Yingjie Zhao ${ }^{*, a}$
}

\begin{abstract}
Two three-dimensional covalent organic frameworks (3D COFs) adopting triptycene as core building blocks were synthesized through $[6+4]$ imine condensation reactions. The stp topology and the " $6+4$ " linking mode were first reported for constructing 3D COFs. A pore size of $4.0 \mathrm{~nm}$ was obtained which is the largest record among the reported 3D COFs. The interpenetration occurred by extending the size of the linkage. The rigid 3D COFs exhibit unique honeycomb nanochannels structures with adjustable pore sizes.
\end{abstract}

Covalent Organic Frameworks (COFs) are crystalline porous polymers with precise structures. ${ }^{1-4}$ As a new generation of organic structures beyond the concept of "atom" and "molecule", the emergence of COFs has greatly attracted the attention of the synthetic chemists. ${ }^{1,5-6}$ COFs have shown promising applications in gas storage and separation, ${ }^{7-8}$ heterogeneous catalysis, ${ }^{9-15}$ optoelectronics, ${ }^{16-22}$ sensing, ${ }^{23-27}$ energy storage, ${ }^{28-29}$ etc benefiting from their unique structures. According to the covalent connectivity, COFs can be categorized as two-dimensional (2D) or three-dimensional (3D) networks..$^{3-6} 2 \mathrm{D}$ COFs with layered structures have been extensively studied. However, 3D COFs with more delicate structures are much less reported. The fundamental reasons could be mainly attributed to the lack of molecular building blocks, complicated network topologies, the difficulties of accurate structural determination and the synthetic challenge. ${ }^{4-6,17,21,30-34}$ Currently, the construction of 3D COFs mainly relies on the tetrahedral linkers possessing $\mathrm{sp}^{3}$-hybridized carbon or silane. ${ }^{30-31,33-38}$ Very few examples reported to date could avoid the tetrahedral geometry in 3D COFs. Roeser, Thomas, and co-workers reported an anionic silicate 3D COF based on hexacoordinate $\left[\mathrm{SiO}_{6}\right]^{2-}$ node and triangular triphenylene building blocks. ${ }^{39}$ Feng and co-workers reported a flexible cyclodextrin-based 3D COF. ${ }^{40}$ Yaghi and co-workers developed a one-dimensional (1D) covalent connectivity, constructed a new series of $3 D$ weaving COF built from the interlocking of $1 \mathrm{D}$ square ribbons. ${ }^{41-43} \mathrm{Up}$ to now, the topological structures of reported 3D COFs are limited to dia (diamond), ${ }^{44} \mathrm{ctn}$ (cubic- $\mathrm{C}_{3} \mathrm{~N}_{4}$ ), ${ }^{32}$ bor (boracite), ${ }^{32}$ pts (platinum sulfide), ${ }^{21,}, 30$ lon (lonsdaleite), ${ }^{45}$ srs (strontium silicide), ${ }^{39} \mathrm{rra}^{40}$ and $\mathrm{ffc}^{46}$.

[a] Prof. Dr. Y. Zhao, Dr. Y. Wang, C. Wu, Q. Pan, W. Hao, H. Liu, Prof. Dr. J. Sun, Prof. Dr. Z. Li

Key Laboratory of Biobased Polymer Materials, Shandong

Provincial Education Department, College of Polymer Science and

Engineering, Qingdao University of Science and Technology, Qingdao, 266042, China

E-mail: yz@qust.edu.cn; zbli@qust.edu.cn

[b] W. Sun, Prof. Dr. J. Sun

College of Chemistry and Molecular Engineering, Beijing National

Laboratory for Molecular Sciences, Peking Universi-ty, Beijing 100871, China

E-mail: junliang.sun@pku.edu.cn

$+\quad$ Y. Wang, C. Wu and W. Sun contributed equally to this work.

Supporting information for this article is given via a link at the end of the document
Developing new building blocks to extend the 3D COFs structures has been an important pursuit of reticular chemistry. ${ }^{1,4-6}$

Although more sophisticated 3D COFs structures have been predicted, to synthesize highly crystalline 3D COFs with new topological structures is still tough challenging. The development of new 3D COFs, especially non-tetrahedral 3D COFs with new topology, is very important for expanding their topological diversity and widening their potential applications. To tackle this challenge, we dedicated to design a new strategy by using trigonal prismatic triptycene and two quadrilateral building blocks including benzene and pyrene to construct conjugated 3D COFs with new topological structures. In this study, two new 3D COFs based on triptycene were obtained through $[6+4]$ imine condensation reactions. The $[6+4]$ connection mode is first reported in constructing 3D COFs which is the first highlight of this work. Detailed structural simulation combined with powder X-ray diffraction (PXRD) measurements demonstrate the 3D COFs adopt stp topology. This new topology which has never been reported for COFs is the second highlight. Thanks to the rigid and stable triptycene core, Trip-COF 1 exhibits the largest pore size of $4.0 \mathrm{~nm}$ among the 3D COFs reported so far which is the third highlight. $^{33}$ Meanwhile, 2-fold interpenetrated structure was observed for Trip-COF 2 via replacing the benzene linking unit with a larger pyrene unit. The longer linkage provides sufficient space. As a result, a possible second interpenetration was achieved. The controllable interpenetration is the fourth highlight of this work. Last but most interestingly, the triptycene based 3D COFs exhibit honeycomb nanochannels structures with different pore sizes according to different linkages. These adjustable unblocked nanochannels with precise pore sizes may show great potential in the area of separation, transport, and delivery system.

The challenge of constructing 3D COFs with novel structures mainly lies in the development of new building blocks, which should be extended along with at least four spatial directions. With this consideration in mind, the rigid triptycene-based building blocks A containing 6 aldehyde groups was selected and synthesized via Suzuki coupling reaction. The triptycene unit has shown great potential in constructing 3D materials. ${ }^{47-50}$ The rigid triangular structure of triptycene enables the formation of hexagonal structure in two-dimensions, while the six aldehydecontaining substituent groups make it possible to construct 3D framework. To adjust the distance of two triptycene units in the direction perpendicular to the hexagonal structure, two amine building blocks $\mathbf{B}$ and $\mathbf{C}$ adopting benzene and pyrene as cores were designed and prepared to connect the trigonal prismatic nodes, respectively. The different sizes of the link units may lead to a different interpenetrated structure. The detailed synthesis was given in the supporting information. Two Trip-COFs were synthesized through the imine condensation reaction of $\mathbf{A}+\mathbf{B}$ and $\mathbf{A}+\mathbf{C}$ in a mixture of 1,2-dichlorobenzene/n-butanol/acetic acid 


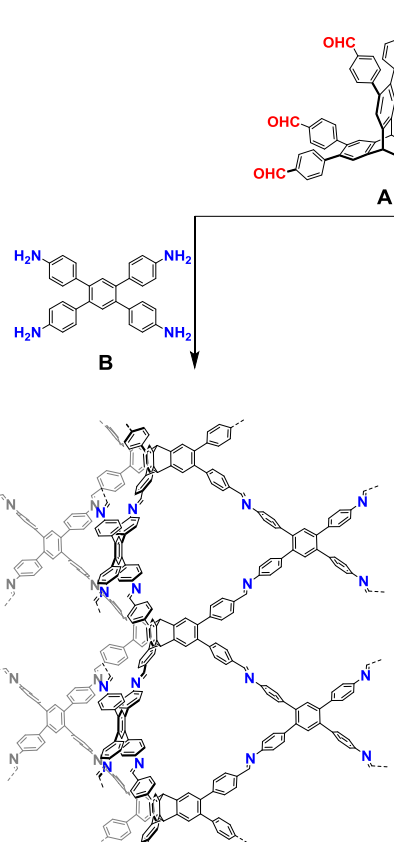

Trip-COF 1
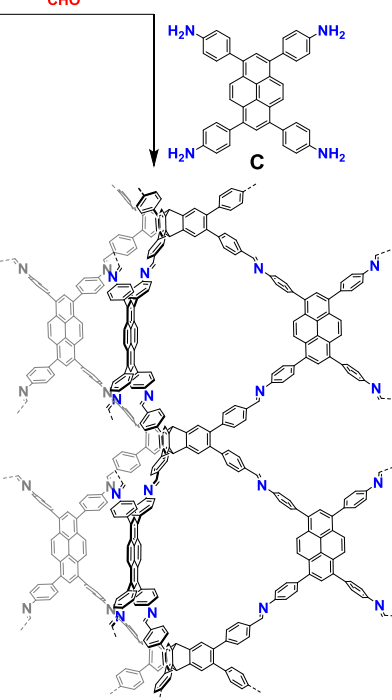

Trip-COF 2

Scheme 1. Synthesis and structures of Trip-COF 1, 2.

(8:2:1) under $120^{\circ} \mathrm{C}$ for 3 days, giving crystalline solids Trip-COF 1 and Trip-COF 2, respectively. The chemical structures of the two 3D COFs were characterized by ${ }^{13} \mathrm{C} \mathrm{CP} / \mathrm{MAS}$ and FT-IR spectra. According to the ${ }^{13} \mathrm{C} C P / M A S$ spectra, the carbon peak appeared at $158 \mathrm{ppm}$ could be attributed to the $\mathrm{C}=\mathrm{N}$ bond and

proved the existence of imine bonds (Figure S1). FT-IR spectra of the Trip-COFs confirmed the formation of the imine linkage, where the $\mathrm{C}=\mathrm{N}$ stretching modes characteristic of the imine were observed at $1624 \mathrm{~cm}^{-1}$ (Figure S2). Thermogravimetric analysis showed that these two Trip-COFs possessed high thermal stability with a decomposition temperature up to $500{ }^{\circ} \mathrm{C}$ (Figure S3). Scanning electron microscopy (SEM) shown in Figure 1 revealed that both of these Trip-COFs exhibited a homogeneous granular-shaped aggregation morphology (for more pictures see ESI, Figure S4, S5). The high crystallinity of the obtained TripCOFs was further confirmed by TEM imaging, where the hexagonal pore of the Trip-COF 1 can be clearly observed accompanied by some 1D channels (Figure 2b, c, d; for more pictures see ESI, Figure S6). For Trip-COF 2, the hexagonal pore can be barely observed, but instead a large amount of $1 \mathrm{D}$ channels which is attributed to the interpenetration (Figure 2f, $\mathrm{g}$, $\mathrm{h}$; for more pictures see ESI, Figure S7).

To evaluate the crystallinity and unit cell parameters and get an in-depth view of the structural information of the obtained TripCOFs, powder X-ray diffraction (PXRD) analysis was performed. As shown in Figure 2, PXRD analysis was conducted on the synthesized Trip-COF 1 crystalline powder (Figure 2a, black line). To characterize the crystal structure, we firstly established a trigonal unit cell with a $P-31 M$ space group (Figure $2 \mathrm{a}$, inset). This is in consideration of the fact that the triptycene unit bears $D_{3 h}$ symmetry and has 6 linking points while the quadruple substituted benzene unit has 4 linking points. Therefore, the proposed " $6+4$ " linking mode of Trip-COF 1 should likely result in a honeycomblike trigonal lattice with hexagonal pores (Figure 2c). Consequently, the $P-31 M$ unit cell of Trip-COF 1 was constructed and optimized by molecular mechanics to exhibit cell parameters of $\mathrm{a}=\mathrm{b}=5.23 \mathrm{~nm}, \mathrm{c}=1.70 \mathrm{~nm}$. This topology was revealed to be
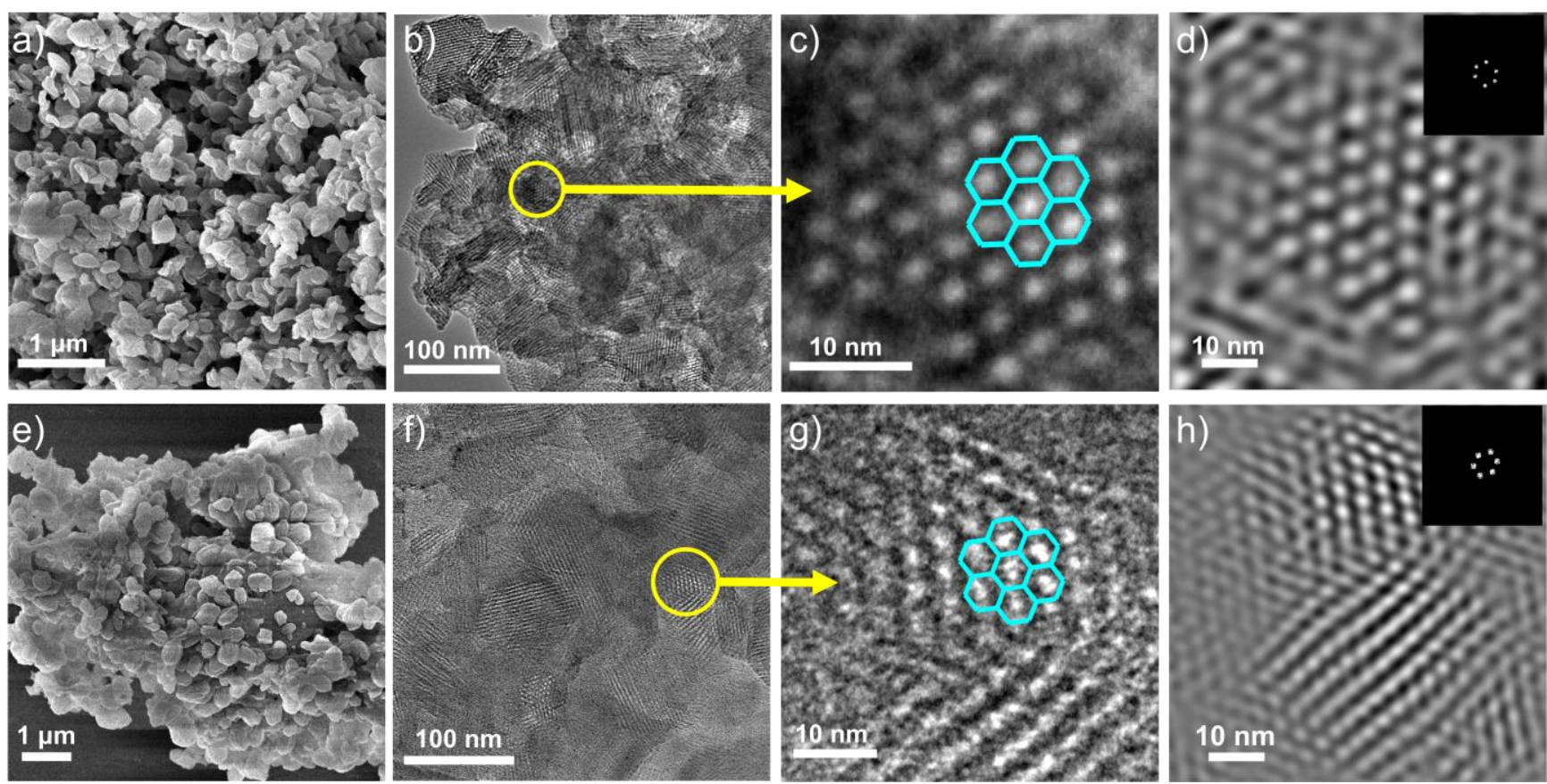

Figure 1. (a) SEM image of Trip-COF 1; (b) TEM image of Trip-COF 1; (c) TEM image of Trip-COF 1 enlarged view of a selected area in panel (b); (d) Fourierfiltered image of selected areas of Trip-COF 1; inset: Fast Fourier Transform (FFT) from the selected areas; for Trip-COF 2 see (e), (f), (g), (h), respectively. 

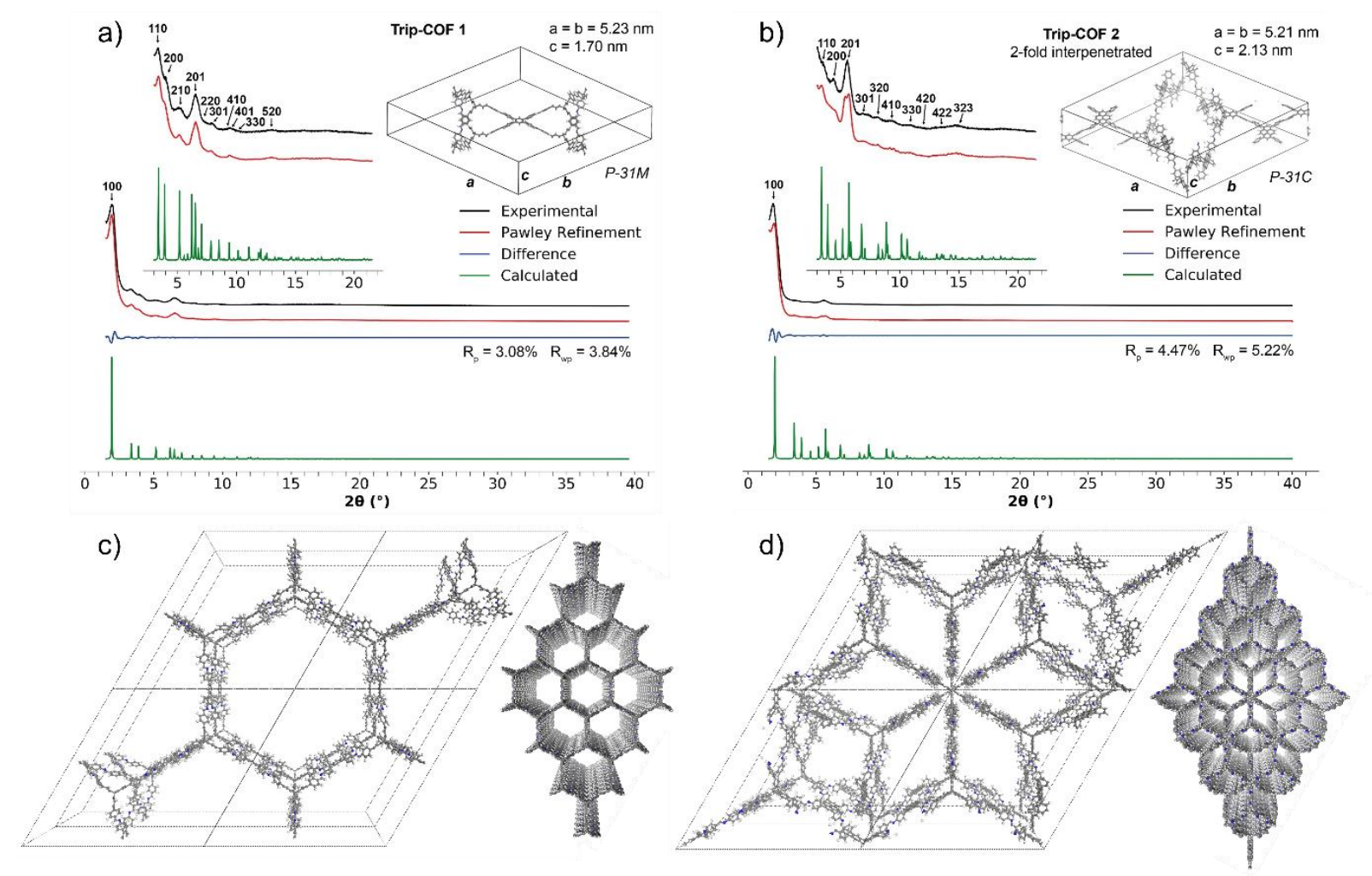

Figure 2. Indexed experimental (black), Pawley refined (red) PXRD patterns with their difference (blue), and the calculated pattern (green) from of noninterpenetrated Trip-COF 1 (a) and Trip-COF 2 with 2-fold interpenetration (b). Inset: zoomed view of detailed PXRD profile without the primary peak; optimized unit cell model of Trip-COF 1 and Trip-COF 2 . Structural representation of Trip-COF 1 (c) and Trip-COF 2 (d). Left: ball-and-stick images; right: space-filling model views perpendicular to 1D channels.

consistent with an existing stp topology which is found for the first time in 3D COFs. Indeed, Pawley refinement of the unit cell revealed minimal difference $\left(R_{p}=3.08 \%\right.$ and $\left.R_{w p}=3.84 \%\right)$, confirming the structure model. Considering the " $6+4$ " linking mode of the 3D Trip-COFs, Trip-COF 2 should have a very similar lattice compared to Trip-COF 1. However, unlike TripCOF 1, Trip-COF 2 has larger space between adjacent triptycene units along the $c$-axis, which thereby generates sufficient space and channels for a possible second interpenetration. Taking this possibility into account, we established both the noninterpenetrated Trip-COF 2 unit cell model characteristic of a $P$ $31 \mathrm{M}$ space group (Figure S8, inset) and the 2-fold interpenetrated Trip-COF 2 unit cell model characteristic of a $P-31 C$ space group (Figure 2b, inset). Geometry optimization and Pawley refinement of both models revealed slightly better agreement from the 2-fold interpenetration (Figure $2 b, R_{p}=4.47 \%$ and $R_{w p}=5.22 \%$ ) rather than no interpenetration (Figure $S 8, R_{p}=4.67 \%$ and $R_{w p}=5.46 \%$ ). Even though this slightly better agreement of 2 -fold interpenetration indeed indicates it is a more likely case, the calculated profiles from both models are highly similar and thus hard to distinguish and clearly conclude.

To address the interpenetration concern of Trip-COF 2, we subsequently conducted $\mathrm{N}_{2}$ adsorption experiments to revealed clear evidence of 2-fold interpenetration by characteristic pore sizes. The surface areas and porosity of Trip-COFs were determined by nitrogen adsorption-desorption analysis at $77 \mathrm{~K}$. As shown in Figure $3 \mathrm{a}$ and $3 \mathrm{c}$, both of the Trip-COFs show type
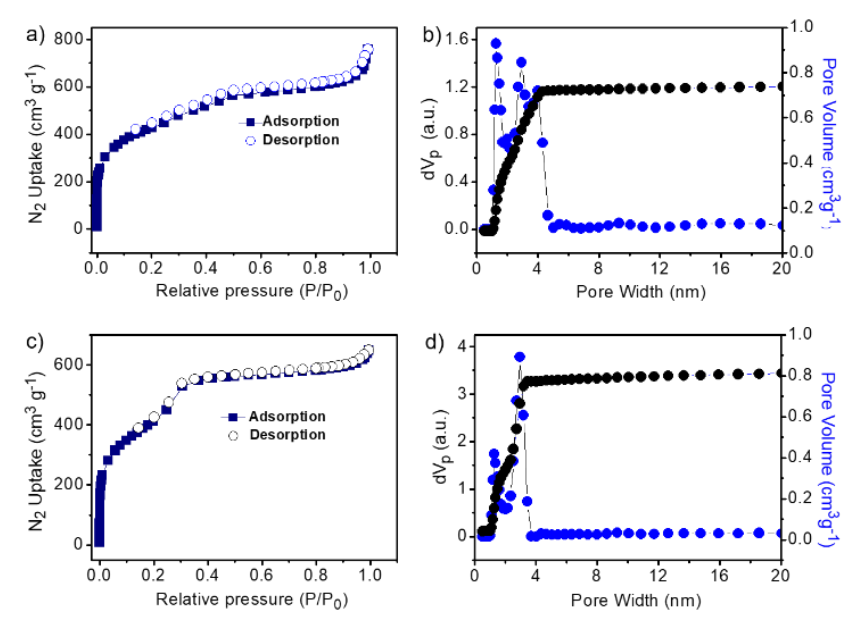

Figure 3. (a, c) $\mathrm{N}_{2}$ adsorption-desorption isotherms (77K) of Trip-COF 1 and 2; (b, d) pore volume and pore size distribution from the quenched solid density functional theory of Trip-COF 1 and 2.

$\mathrm{I}$ isotherm with a sharp uptake under low-pressure stage $\left(\mathrm{P} / \mathrm{P}_{0}<\right.$ 0.05 ), followed by a second step at about $P / P_{0}=0.2$, indicating the mesoporous characteristics of the Trip-COFs. The BrunauerEmmett-Teller (BET) surface area of Trip-COF 1 and Trip-COF 2 were obtained as $S_{B E T}=1473 \mathrm{~m}^{2} \mathrm{~g}^{-1}$ and $1624 \mathrm{~m}^{2} \mathrm{~g}^{-1}$, respectively. The total pore volume was calculated to be $V_{p}=1.17 \mathrm{~cm}^{3} \mathrm{~g}^{-1}$ and 
$1.00 \mathrm{~cm}^{3} \mathrm{~g}^{-1}$ at $\mathrm{P} / \mathrm{P}_{0}=0.99$, respectively. As shown in Figure $3 \mathrm{~b}$, the pore size distribution curve clearly shows three different pore limiting diameters (PLD) for Trip-COF 1 (12.6 A, $29.6 \AA$ A and 39.9 $\AA)$. According to the simulation results of the pore sizes, the theoretical PLD is $42.0 \AA$, which well correlates to the experimentally determined $39.9 \AA$ with $E_{r}<5 \%$. As a contrast, Trip-COF 2 displays only two different mesopores with PLD values of $12.6 \AA$ and $29.3 \AA$. The largest PLD is missing compared with Trip-COF 1 due to the 2-fold-interpenetrated structure which provides clear evidence for the formation of the interpenetration. The theoretical PLD is $15 \AA$ which is also consistent with the experimental result (12.6 ̊).

In summary, we report the design and synthesis of novel 3D COFs crystallizing in an elaborate topological structure. Triptycene as a new linkage was first used in constructing 3D COFs. Two unique 3D crystalline structures with controllable sizes of $1 D$ nanochannels were obtained via the $[6+4]$ imine condensation reactions between the triptycene with 6 aldehyde groups and building blocks containing 4 amine groups. The stp topology of the two 3D COFs has never been reported before. This work provides a new choice to avoid the tetrahedral building blocks for constructing 3D COFs and expanding their diversities. The triptycene as a new linkage will provide more opportunities in the design and synthesis of more elaborate crystalline structures and extend the topologies of 3D COFs. The new [6+4] linking mode together with the stp topology are both first reported for 3D COFs. The controllable interpenetration in constructing COFs materials is also a breakthrough which may provide a fundamental guide for studying the complex structure of COFs. More linkages with different sizes and lengths such as perylene derivatives are being tried to get more complicated multipleinterpenetrated structures and conclude the regularity. The unique nanochannel structures with large pore sizes endow the materials' potential application in catalysis, separation, and adsorption of large molecules. In addition, the $\pi$ electron delocalization along the wall of the honeycomb 1D nanochannels brings great potential for optoelectronic application.

\section{Acknowledgements}

The work was supported by the National Natural Science Foundation of China (21905150, 51722302 and 21674054), China Postdoctoral Science Foundation (2019M652338).

\section{Keywords:}

[1] C. S. Diercks, O. M. Yaghi, Science 2017, 355, eaal1585

[2] S. Y. Ding, W. Wang, Chem. Soc. Rev. 2013, 42, 548-568.

[3] N. Huang, P. Wang, D. Jiang, Nat. Rev. Mater. 2016, 1, 16068.

[4] K. Geng, T. He, R. Liu, S. Dalapati, K. T. Tan, Z. Li, S. Tao, Y. Gong, Q. Jiang, D. Jiang, Chem. Rev. 2020, 10.1021/acs.chemrev.9b00550

[5] S. J. Lyle, P. J. Waller, O. M. Yaghi, Trends in Chemistry 2019, 1, 172184

[6] O. M. Yaghi, Mol. Front. J. 2019, 03, 66-83.

[7] H. Fan, A. Mundstock, A. Feldhoff, A. Knebel, J. Gu, H. Meng, J. Caro, J. Am. Chem. Soc. 2018, 140, 10094-10098.
[8] Q. Gao, X. Li, G.-H. Ning, H.-S. Xu, C. Liu, B. Tian, W. Tang, K. P. Loh, Chem. Mater. 2018, 30, 1762-1768.

[9] S. Y. Ding, J. Gao, Q. Wang, Y. Zhang, W. G. Song, C. Y. Su, W. Wang J. Am. Chem. Soc. 2011, 133, 19816-19822.

[10] X. Han, Q. Xia, J. Huang, Y. Liu, C. Tan, Y. Cui, J. Am. Chem. Soc. 2017, 139, 8693-8697.

[11] S. Lin, C. S. Diercks, Y.-B. Zhang, N. Kornienko, E. M. Nichols, Y. Zhao, A. R. Paris, D. Kim, P. Yang, O. M. Yaghi, C. J. Chang, Science 2015, 349, 1208.

[12] S. Lu, Y. Hu, S. Wan, R. McCaffrey, Y. Jin, H. Gu, W. Zhang, J. Am. Chem. Soc. 2017, 139, 17082-17088.

[13] Q. Sun, B. Aguila, J. Perman, N. Nguyen, S. Ma, J. Am. Chem. Soc. 2016, 138, 15790-15796.

[14] P.-F. Wei, M.-Z. Qi, Z.-P. Wang, S.-Y. Ding, W. Yu, Q. Liu, L.-K. Wang, H.-Z. Wang, W.-K. An, W. Wang, J. Am. Chem. Soc. 2018, 140, 46234631.

[15] H. Xu, J. Gao, D. Jiang, Nat. Chem. 2015, 7, 905-912.

[16] S. Dalapati, E. Jin, M. Addicoat, T. Heine, D. Jiang, J. Am. Chem. Soc. 2016, 138, 5797-5800.

[17] H. Ding, J. Li, G. Xie, G. Lin, R. Chen, Z. Peng, C. Yang, B. Wang, J. Sun, C. Wang, Nat. Commun. 2018, 9, 5234.

[18] S. Haldar, D. Chakraborty, B. Roy, G. Banappanavar, K. Rinku, D. Mullangi, P. Hazra, D. Kabra, R. Vaidhyanathan, J. Am. Chem. Soc 2018, 140, 13367-13374.

[19] H. Li, J. Chang, S. Li, X. Guan, D. Li, C. Li, L. Tang, M. Xue, Y. Yan, V. Valtchev, S. Qiu, Q. Fang, J. Am. Chem. Soc. 2019, 141, 13324-13329.

[20] X. Li, Q. Gao, J. Wang, Y. Chen, Z. H. Chen, H. S. Xu, W. Tang, K. Leng G. H. Ning, J. Wu, Q. H. Xu, S. Y. Quek, Y. Lu, K. P. Loh, Nat. Commun. 2018, 9, 2335.

[21] G. Lin, H. Ding, D. Yuan, B. Wang, C. Wang, J. Am. Chem. Soc. 2016, 138, 3302-3305.

[22] C. Wu, Y. Liu, H. Liu, C. Duan, Q. Pan, J. Zhu, F. Hu, X. Ma, T. Jiu, Z. Li, Y. Zhao, J. Am. Chem. Soc. 2018, 140, 10016-10024.

[23] L. Ascherl, E. W. Evans, J. Gorman, S. Orsborne, D. Bessinger, T. Bein R. H. Friend, F. Auras, J. Am. Chem. Soc. 2019, 141, 15693-15699.

[24] D. Bessinger, L. Ascherl, F. Auras, T. Bein, J. Am. Chem. Soc. 2017, 139 12035-12042.

[25] S.-Y. Ding, M. Dong, Y.-W. Wang, Y.-T. Chen, H.-Z. Wang, C.-Y. Su, W. Wang, J. Am. Chem. Soc. 2016, 138, 3031-3037.

[26] X. Liu, D. Huang, C. Lai, G. Zeng, L. Qin, H. Wang, H. Yi, B. Li, S. Liu, M. Zhang, R. Deng, Y. Fu, L. Li, W. Xue, S. Chen, Chem. Soc. Rev. 2019, 48, 5266-5302.

[27] X. Wu, X. Han, Q. Xu, Y. Liu, C. Yuan, S. Yang, Y. Liu, J. Jiang, Y. Cui, J. Am. Chem. Soc. 2019, 141, 7081-7089.

[28] C. R. DeBlase, K. E. Silberstein, T.-T. Truong, H. D. Abruña, W. R. Dichtel, J. Am. Chem. Soc. 2013, 135, 16821-16824.

[29] Z. Lei, Q. Yang, Y. Xu, S. Guo, W. Sun, H. Liu, L. P. Lv, Y. Zhang, Y. Wang, Nat. Commun. 2018, 9, 576.

[30] G. Lin, H. Ding, R. Chen, Z. Peng, B. Wang, C. Wang, J. Am. Chem. Soc. 2017, 139, 8705-8709.

[31] C. Gao, J. Li, S. Yin, G. Lin, T. Ma, Y. Meng, J. Sun, C. Wang Angew .Chem. Int. Ed. 2019, 58, 9770-9775.

[32] H. M. El-Kaderi, J. R. Hunt, J. L. Mendoza-Cortes, A. P. Cote, R. E. Taylor, M. O'Keeffe, O. M. Yaghi, Science 2007, 316, 268-272.

[33] Y. Wang, Y. Liu, H. Li, X. Guan, M. Xue, Y. Yan, V. Valtchev, S. Qiu, Q. Fang, J. Am. Chem. Soc. 2020, 142, 3736-3741.

[34] C. Gao, J. Li, S. Yin, J. Sun, C. Wang, J. Am. Chem. Soc. 2020, 142 3718-3723.

[35] X. Guan, Y. Ma, H. Li, Y. Yusran, M. Xue, Q. Fang, Y. Yan, V. Valtchev, S. Qiu, Fast, J. Am. Chem. Soc. 2018, 140, 4494-4498.

[36] J. Huang, X. Han, S. Yang, Y. Cao, C. Yuan, Y. Liu, J. Wang, Y. Cui, J. Am. Chem. Soc. 2019, 141, 8996-9003.

[37] T. Ma, J. Li, J. Niu, L. Zhang, A. S. Etman, C. Lin, D. Shi, P. Chen, L.-H Li, X. Du, J. Sun, W. Wang, J. Am. Chem. Soc. 2018, 140, 6763-6766.

[38] S. Yan, X. Guan, H. Li, D. Li, M. Xue, Y. Yan, V. Valtchev, S. Qiu, Q. Fang, J. Am. Chem. Soc. 2019, 141, 2920-2924. 
[39] O. Yahiaoui, A. N. Fitch, F. Hoffmann, M. Fröba, A. Thomas, J. Roeser, J. Am. Chem. Soc. 2018, 140, 5330-5333.

[40] Y. Zhang, J. Duan, D. Ma, P. Li, S. Li, H. Li, J. Zhou, X. Ma, X. Feng, B. Wang, Angew .Chem. Int. Ed. 2017, 56, 16313-16317.

[41] Y. Liu, Y. Ma, Y. Zhao, X. Sun, F. Gándara, H. Furukawa, Z. Liu, H. Zhu, C. Zhu, K. Suenaga, P. Oleynikov, A. S. Alshammari, X. Zhang, O Terasaki, O. M. Yaghi, Science 2016, 351, 365.

[42] Y. Liu, C. S. Diercks, Y. Ma, H. Lyu, C. Zhu, S. A. Alshmimri, S. Alshihri, O. M. Yaghi, J. Am. Chem. Soc. 2019, 141, 677-683.

[43] Y. Liu, Y. Ma, J. Yang, C. S. Diercks, N. Tamura, F. Jin, O. M. Yaghi, J. Am. Chem. Soc. 2018, 140, 16015-16019.

[44] F. J. Uribe-Romo, J. R. Hunt, H. Furukawa, C. Klöck, M. O’Keeffe, O. M. Yaghi, J. Am. Chem. Soc. 2009, 131, 4570-4571.

[45] T. Ma, E. A. Kapustin, S. X. Yin, L. Liang, Z. Zhou, J. Niu, L.-H. Li, Y. Wang, J. Su, J. Li, X. Wang, W. D. Wang, W. Wang, J. Sun, O. M. Yaghi, Science 2018, 361, 48

[46] Y. Lan, X. Han, M. Tong, H. Huang, Q. Yang, D. Liu, X. Zhao, C. Zhong, Nat. Commun. 2018, 9, 5274.

[47] Z. Chen, P. Li, R. Anderson, X. Wang, X. Zhang, L. Robison, L. R. Redfern, S. Moribe, T. Islamoglu, D. A. Gómez-Gualdrón, T. Yildirim, J. F. Stoddart, O. K. Farha, Science 2020, 368, 297.

[48] P. Li, P. Li, M. R. Ryder, Z. Liu, C. L. Stern, O. K. Farha, J. F. Stoddart, Angew. Chem. Int. Ed. 2019, 58, 1664-1669.

[49] D. Reinhard, F. Rominger, M. Mastalerz, J. Org. Chem. 2015, 80, $9342-$ 9348.

[50] D. Reinhard, W.-S. Zhang, Y. Vaynzof, F. Rominger, R. R. Schröder, M. Mastalerz, Chem. Mater. 2018, 30, 2781-2790. 
Yuancheng Wang,,$^{a,+}$ Chenyu Wu, ${ }^{a,+}$ Wenjia Sun, ${ }^{b,+}$ Qingyan Pan, ${ }^{a}$ Wenbo

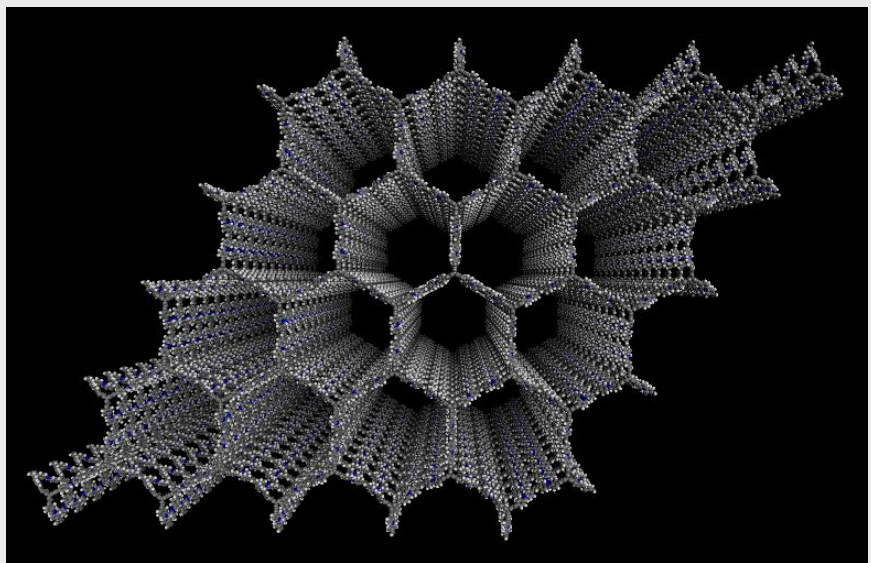
Hao, ${ }^{a}$ Hui Liu, ${ }^{a}$ Jing Sun, ${ }^{a}$ Zhibo Li, ${ }^{*}, a$ Junliang Sun, ${ }^{*}, b$ and Yingjie Zhao, ${ }^{*}$

Page No. - Page No.

Triptycene-based Three-Dimensional Covalent Organic Frameworks with stp Topology of Honeycomb Structure

Two triptycene based 3D COFs with stp topology and the "6 + 4" linking mode was synthesized. The 3D COFs exhibit honeycomb nanochannels structures with different pore sizes according to different linkages. 